Revista General de Información y Documentación ISSN: 1132-1873

http://dx.doi.org/10.5209/rgid.72821

\title{
Implementación de un plan anual de metas para la mejora de la producción científica en una universidad colombiana. Aspectos positivos y negativos
}

\author{
Pedro Vázquez-Miraz ${ }^{1}$; Marcos Ricardo Posada Llorente ${ }^{2}$
}

Recibido: 7 de julio de 2020 / Aceptado: 15 de noviembre de 2020

Resumen. Se presenta en la siguiente investigación un análisis descriptivo pormenorizado de la producción científica de alto impacto (definida ésta como toda investigación indexada en la base de datos Scopus) de una universidad privada colombiana, la Universidad Tecnológica de Bolívar, durante el período temporal 2017-2019. El objetivo de este trabajo es dilucidar los aspectos positivos y negativos de la implementación de un plan de metas anual relacionado con la producción científica del profesorado de planta de esta entidad educativa superior durante su primer año de vigencia (año 2019). Para ello, se revisó la información de la base de datos Scopus en función de diversas variables tales como el tipo de producto, número de autores, área temática... y su evolución temporal en esos tres años, haciendo también una comparativa con el resto de las universidades de su entorno más cercano. Aunque se logró superar con creces la meta global impuesta (superar el centenar de productos), la asignación de cuotas de producción científica al profesorado profundizó otras tendencias ya existentes en esta universidad como la extrema dependencia del trabajo de ciertos departamentos de ingeniería y la preferencia por publicar conference proceedings y no libros, capítulos de libro o revistas.

Palabras clave: Producción científica; investigación; gestión del conocimiento; universidades colombianas; Cartagena de Indias

\section{[en] Implementation of an Annual Goal's Plan for the improvement of scientific production in a Colombian university. Positives and negatives aspects}

\begin{abstract}
1 Universidad Tecnológica de Bolívar Facultad de CC.SS. y humanidades.
E-mail: pvasquez@utb.edu.co

2 Universidad Tecnológica de Bolívar Facultad de CC.SS. y humanidades.

E-mail: mposada@utb.edu.co
\end{abstract}

Abstract. We present in the following investigation, a detailed descriptive analysis of the high-impact scientific production (defined this as all research indexed in the Scopus) database of a Colombian private university, the Universidad Tecnológica de Bolívar, during the period between 2017 - 2019. The purpose of this paper is to clarify the positives and negatives aspects of the implementation of an Annual Goal's Plan, related to the scientific production of the teaching staff of this University during the first year of validity (2019). For it, it was reviewed the information of the Scopus database according 
with several variables such as the kind of product, number of authors, thematic area... and its temporal evolution in these three years, also making a comparison with the rest of universities of its closest environment. Although, the institution was able to overcome by far the goal set (surpass a hundred products), the allocation of scientific production quotas for teachers deepened other trends already present in this university, such as the extreme dependence on work of certain Engineering departments, and the preference for publishing conference proceedings instead of books, book's chapters or journals. Keywords: Scientific Production; research; knowledge management; Colombian universities; Cartagena de Indias.

Sumario. 1. Introducción. 2. Contexto histórico y social de la investigación. 3. La propuesta del Plan de Metas Anual de la Universidad Tecnológica de Bolívar. 4. Metodología. 5. Resultados. 6. Discusión de resultados y conclusiones. 7. Referencias bibliográficas. 8. Anexo.

Cómo citar: Vázquez-Miraz, P.; Posada Llorente, M. R. (2020) Implementación de un plan anual de metas para la mejora de la producción científica en una universidad colombiana. Aspectos positivos y negativos, en Revista General de Información y Documentación 30 (2), 457-471.

\section{Introducción}

La producción científica es uno de los pilares fundamentales que justifica el ser de cualquier universidad y desde esta perspectiva el estudiar los insumos de las propias instituciones educativas ha fomentado numerosas pesquisas (Rueda-Barrios y Rodenes-Adam, 2016), si bien, como afirmaban Avital y Collopy (2001, citados en Rueda-Barrios y Rodenes-Adam, 2016) y Alarcón-Quinapanta et al. (2019) no hay un único factor explicativo que explique el alto rendimiento en el campo científico, al estar involucrados en la comprensión de esta variable factores profesionales, institucionales, económicos, culturales, etc.

Las universidades, entendidas como los eslabones fundamentales para la creación de nuevo conocimiento, se caracterizan en Colombia por poseer un bajo nivel de eficiencia investigativa (Bayona-Rodríguez, Bedoya y Sánchez, 2018). Una situación particular muy preocupante puesto que casi la totalidad de la investigación científica colombiana se gesta en este tipo de instituciones (Bayona-Rodríguez, et al., 2018). Es por eso, que se requieren acciones y mecanismos para conseguir una mayor calidad y cantidad investigativa, indistintamente del área que cada profesor o investigador trabaje, pues la producción en ciencia se asocia irremediablemente al capital intelectual de las naciones, mejorándose así la gestión del conocimiento o la competitividad entre otros factores (Alarcón-Quinapanta et al., 2019).

Son claras y están bien fundamentadas muchas de las críticas de la comunidad académica hacia el deber de elaborar y publicar documentos científicos revisados por pares anónimos, el célebre axioma anglosajón de publish or perish que tanto perjudica al mundo hispanoparlante (Suárez-Ortega, García-Mingo y Ruiz-San Román, 2012); advirtiendo muchos maestros que en los tiempos actuales la docencia superior y su independencia se está mercantilizando de forma desmedida (Montes y Mendoza, 2018; Puentes-Cala, 2019) en aras del logro de unos meros indicadores manejados por empresas privadas (Giménez-Toledo, 2014; Caldera-Serrano, 2018), señalando también que la formación de los estudiantes cada vez tiene una menor 
relevancia en las universidades frente a la omnímoda producción científica (Montes y Mendoza, 2018). Como comentan lacónicamente Martínez-Dueñas y Arias Ocampo $^{3}$ (2017, p. 12):

Quizás sí perdimos el rumbo, pero eso no quiere decir que todo este perdido (...). Desde la lógica del "publish or perish", la revista [en referencia a la publicación que gestionan estos dos autores] posee una dinámica de citación que hace que mantengamos el compromiso de seguirla editando, tratando de hacernos un espacio en la lógica de las políticas transnacionales de la publicación científica que hemos asumido sin cuestionamiento alguno.

De todas maneras, consideraríamos que estos valiosos argumentos nunca pueden ser una excusa para justificar el extremo opuesto; la escasa capacidad investigadora de alto impacto de muchas corporaciones educativas de América Latina y el Caribe (siendo Brasil el referente de esta región a seguir), a pesar de una mejoría global respecto el pasado siglo (Santa y Herrero-Solana, 2010). Una situación que ha afectado más especialmente a Colombia (Rodríguez-Morales, Culquichicón-Sánchez y GilRestrepo, 2016). Hecho que se explicara, entre otros factores, a la fuerte apuesta de los investigadores nacionales por las revistas locales y no por las internacionales (MazMachado, Jiménez-Fanjul y Villarraga, 2016). Si bien, teniendo en cuenta el magro presupuesto otorgado a la ciencia de este país americano, se podría considerar que la ciencia colombiana ha sido más eficiente que el de otras naciones de su entorno al manejar ésta unos reducidos recursos económicos (Gómez-Velasco, JiménezGonzález, Rodríguez-Gutiérrez y Romero-Torres, 2020).

Es por todo lo dicho anteriormente que presentamos un estudio de una universidad colombiana, la Universidad Tecnológica de Bolívar (UTB), que en el año 2019 implementó una nueva forma de evaluación docente con el objetivo de aumentar la producción científica. El interés y novedad de esta propuesta radicaría en que en este documento no solo se ha plasmado una mera evolución de estadísticas relacionadas con la producción científica de una universidad; pues el objetivo principal de este artículo fue el de dilucidar la eficacia de este cambio administrativo y destacar sus fortalezas y sus debilidades con datos tangibles como son los datos relacionados a la cantidad y a la calidad de documentos científicos de alto impacto que ha generado anualmente una institución de educación superior.

En los últimos años, la UTB ha modificado radicalmente su visión corporativa a partir del año 2018 con un cambio de gobierno rectoral, al haberse pasado de considerarse una universidad de docencia con un interés secundario en el campo de la investigación, a asumir el rol de ser una entidad destinada a partes iguales a la docencia y a la investigación. Es por ello por lo que los profesores de planta de esta universidad, antiguamente clasificados informalmente en función de su interés en la investigación o en la docencia, desde el 2019 debían desempeñar ineludiblemente actividades de investigación.

3 Editor y asistente de la revista colombiana Jangwa Pana en esa época. 


\section{Contexto histórico y social de la investigación}

La historia de la educación universitaria en la Región Caribe colombiana comenzó en el año de 1806 con la aprobación mediante Cédula Real del Colegio Universidad de San Pedro Apóstol en el municipio bolivarense de Mompox; iniciando la prestación del servicio educativo en el año de 1809 (Arango-Soto, 2010). En lo referente a la ciudad de Cartagena de Indias, la primera institución universitaria fundada fue la Universidad de Cartagena, de carácter estatal y creada por el Congreso de la República de Colombia mediante el decreto del 06 de octubre de 1827 bajo el nombre inicial de Universidad del Magdalena, comenzando a funcionar en 1828 con la escuela de Filosofía y Letras (Ferro-Bayona y Amarís, 1991).

Habría que esperar aproximadamente un siglo y medio para ser testigos del nacimiento de la primera universidad de carácter privado en esta urbe. Esta institución fue la Universidad Tecnológica de Bolívar, fundada el 05 de agosto de 1970 bajo el nombre de Corporación Universitaria Tecnológica de Bolívar, comenzando sus clases el 03 de marzo de 1971, ofertando desde sus inicios los programas de economía e ingenierías eléctrica, mecánica e industrial, sosteniendo un ritmo continuo de crecimiento en el número de programas y de estudiantes. Para el año 2003, este centro recibe por parte del Ministerio de Educación Nacional de Colombia el reconocimiento como universidad, adoptando su nombre actual (Ripoll, 2011).

El surgimiento de este nuevo agente educativo en Cartagena no fue un hecho aislado, pues en la década de los setenta del siglo XX (periodo en el que surgió la Universidad Tecnológica de Bolívar), la Región Caribe colombiana fue testigo del surgimiento de varias universidades; estando la mayoría ubicadas en la ciudad vecina de Barranquilla, tal y cómo describe Viloria-De La Hoz (2006):

[...aparecieron respectivamente] la Corporación Universitaria de la Costa - CUC (1971), Universidad Simón Bolívar (1972) y Universidad Metropolitana (1973); en Montería se creó la Corporación Universitaria del Sinú (1977) y en Santa Marta se abrió una sede de la Universidad Cooperativa de Colombia (1980). En las siguientes dos décadas se amplió acelerada y desordenadamente la oferta educativa en toda la región Caribe, siendo las universidades privadas las que tuvieron mayor crecimiento (pp. 10-11).

La ciudad de Cartagena no iba a ser la excepción con respecto al surgimiento acelerado de varias instituciones de educación superior a partir de los años ochenta del pasado siglo. Dentro de las entidades de educación que consideramos más relevantes de esta población caribeña, podemos mencionar aquellas que registran alguna producción intelectual en la base de datos Scopus (con datos de junio del 2020) como lo son la Fundación Universitaria Tecnológico Comfenalco (1984), la Corporación Universitaria Rafael Núñez (1985) y la Universidad de San Buenaventura (1992, como seccional autónoma). Sin hacer ninguna distinción, debemos indicar que este crecimiento se mantuvo con el paso de los años y en la actualidad, la ciudad cuenta con un total de 13 universidades domiciliadas, de acuerdo con las cifras más recientes del Ministerio de Educación Nacional de Colombia (información consultada en junio del año 2020). 
Por último, dentro del específico contexto de la educación universitaria en Colombia, debemos resaltar la figura de la Acreditación Institucional, entendida esta como el reconocimiento de carácter oficial otorgado a aquellas instituciones de educación superior que de manera voluntaria se someten a un proceso de auto evaluación y logran demostrar los estándares de calidad exigidos. El Sistema Nacional de Acreditación en Colombia fue establecido por el Art. 53 de la Ley 30 de 1992, que regula la educación en esta nación americana.

La Universidad Tecnológica de Bolívar comenzó su proceso de autoevaluación con fines de Acreditación en el año 2002, obteniendo el reconocimiento en el año 2011 por un término de cuatro años, el cual se ha renovado de manera continua hasta los tiempos actuales. Es de destacar que la UTB fue la primera institución universitaria en obtener dicho logro en la ciudad de Cartagena. El objetivo de obtener la Acreditación Institucional y conservarla, ha sido determinante para el fomento de la investigación y el incentivo al aumento de publicaciones científicas, toda vez que uno de los factores más importantes de acreditación lo constituye, sin lugar a dudas, la investigación científica.

\section{La propuesta del Plan de Metas Anual de la Universidad Tecnológica de Bolívar}

El Plan de Metas Anual (PMA) de la Universidad Tecnológica de Bolívar (UTB) fue una propuesta de evaluación del desempeño profesoral diseñada por el equipo de Excelencia Docente y Apoyo al Aprendizaje (EXDA), órgano creado por la nueva administración de la institución, con el fin de sustituir el anterior modelo de evaluación del desempeño docente basado en un simple informe cualitativo anual (denominado Workplan), a través del cual los docentes de planta debían señalar al comienzo del semestre, como sería la distribución en cada semana del tiempo asignado por la institución para el cumplimiento de sus funciones.

Esto último se aplicaba segregando de manera porcentual la carga semanal del profesor de planta (40 horas los de tiempo completo) en cuatro áreas básicas (docencia, investigación, gestión académica y extensión). Así pues, por ejemplo, un docente sin producción científica incidía más en su informe en las horas impartidas de clase, en la preparación de estas y en las asesorías realizadas a sus alumnos mientras que otro compañero más versado en la investigación y que consiguió publicar algún documento científico ese año, se asignaba más horas en el apartado investigativo.

Conocidas las debilidades de esta evaluación cualitativa, resumidas fundamentalmente por su imposibilidad de presentar un resultado desfavorable al facilitar sobre manera la justificación del trabajo docente, el nuevo equipo rectoral diseñó (sin la participación del cuerpo profesoral) una evaluación cuantitativa, tipificada en una escala de 1 (mínimo) a 5 (máximo), basada en una serie de objetivos relacionados con las áreas de investigación y producción intelectual, gestión académica y extensión (Anexo). Este instrumento fue socializado al cuerpo docente a finales del año 2018 e impuesto jerárquicamente, siendo este cambio recibido con inquietud por parte del cuerpo profesoral al verse éste como una general precarización 
de su puesto de trabajo (Castillo y Moré, 2016), además del acto específico de no haberse ofrecido la posibilidad de realizar modificaciones de mejora al documento ${ }^{4}$.

A partir del año 2019, todos los docentes de planta de la UTB (de tiempo completo) debían cumplir tres actividades (a excepción de los de medio tiempo y los que tuvieran asignado un cargo administrativo, que tendrían solamente una) exigiéndose el publicar (o conseguir la aceptación) de un documento científico (no válido para el cumplimiento de esta meta las reseñas, las cartas al editor u otros productos similares) en Scopus o la Web of Science (WoS) ${ }^{5}$ mientras que los profesores con título de doctor tenían como objetivo obligatorio el conseguir dos productos de estas mismas categorías. Los otros dos objetivos eran de libre elección del profesor (pudiendo éste seleccionar varias veces una misma meta hasta alcanzar su cuota e incluso solicitar metas adicionales en aras de demostrar su productividad laboral), estando siempre este proceso supervisado por los directores de los programas y los decanos de cada facultad. $\mathrm{Al}$ momento de escoger las 3 metas anuales, los docentes debían suscribir un documento compromisorio con la institución.

Así pues, este PMA equivale al 50\% de la evaluación docente mientras que el resto de la puntuación viene dado por otros tres insumos. El promedio de las calificaciones dadas al profesor por los estudiantes de las materias impartidas a través de una encuesta anónima (un 20\%, siendo esta categoría el antiguo apartado de docencia), la valoración asignada por la decanatura (otro 20\%) y una autoevaluación de desempeño (el 10\% restante). Evidentemente el resultado obtenido en el PMA tenía un impacto directo en aspectos laborales futuros muy relevantes para el profesorado, tales como la posibilidad o negación de ascensos en el escalafón salarial o incluso la posibilidad de no renovación de contratos o el fin de estos por baja productividad.

\section{Metodología}

Para la realización de esta investigación se ha empleado un diseño descriptivo, en el que básicamente se revisaron las características primordiales de la producción científica de alto impacto de la UTB. Para ello se manejó la plataforma Scopus y se hizo una comparativa entre la investigación alcanzada en su seno (ignorándose expresamente el resto de los trabajos académicos presentes en otras bases de datos) en el primer año en el que estuvo vigente el PMA (2019) y los dos años anteriores

4 Una de las mayores críticas docentes hechas al PMA fue todo lo relativo a la rígida evaluación de esta medición, pues ésta se planteaba de manera dicotómica ( 1 = No cumplido / 5 = cumplido). Esta inflexible estructura se evidenciaba en mayor grado en el objetivo obligatorio de conseguir una publicación (o aceptación) de un documento en Scopus y/o WoS debido a los alargados plazos temporales que requiere un peer review y otras casuísticas asociadas a este proceso.

5 Paradójicamente el publicar en revistas catalogadas en el índice Emerging Sources Citation Index (ESCI) de la WoS, indicador manejado por investigadores para identificar la producción científica en Colombia (MuñozÑungo, Rodríguez-Faneca y Gutiérrez-Rubio, 2020) no fue aceptado por las instancias evaluadoras de la universidad, a pesar de que el PMA no especificaba en ningún momento los índices en donde se debía publicar. 
como indicadores de los antecedentes más relevantes a la implementación de este instrumento de medición del desempeño profesoral.

Se trabajó en exclusiva con la plataforma Scopus, obviándose otras bases científicas de gran reconocimiento como la Web of Science (Mongeon y Paul-Hus, 2016; Vélez-Cuartas, Lucio-Arias y Leydesdorff, 2016) o de carácter más regional y local como SciELO (Maz-Machado et al., 2016; Vélez-Cuartas et al., 2016) o Publindex (Rodríguez, Naranjo y González, 2015), al ser el objetivo prioritario de esta institución educativa el superar el centenar de productos en la primera herramienta para así poder estar escalafonado en rankings internacionales de universidades como el prestigioso QS World Universiy Ranking (Torres-Samuel et al., 2018) y así aumentar su reconocimiento social en el competido contexto de América Latina, específicamente en la Región del Caribe colombiano, para también conseguir una mayor proyección global.

Además de analizar y comparar la producción científica total de esta universidad colombiana en estos tres respectivos años (contando los productos depredadores y sin ellos), la documentación revisada fue catalogada en función de las siguientes variables:

- Número total de productos científicos y su tipología (libros y capítulos de libro / artículo y revisión / congresos científicos / otros).

- Área de conocimiento $^{6}$ y procedencia de la autoría por facultad y departamento.

- Fuentes principales donde se ha conseguido publicar ${ }^{7}$ (sin distinción entre revistas de acceso abierto y acceso previo pago).

- Idioma utilizado para la escritura del documento.

Adicionalmente, para enriquecer nuestros datos y evidenciar mejor los efectos del PMA de la UTB en su producción científica de alto impacto, se ha realizado una comparativa de esta misma variable con el resto de las universidades de la ciudad de Cartagena de Indias que aparecen registrada en Scopus: [Universidad de Cartagena (pública), Universidad San Buenaventura (privada), Fundación Universitaria Tecnológico Comfenalco (privado) y Corporación Universitaria Rafael Núñez (privado)] durante el período temporal 2017-2020.

\section{Resultados}

Dentro del maremágnum de cifras y resultados hallados en Scopus (junio del 2020), indicamos que lo más destacable de lo revisado fue el incremento continuo de la producción científica registrada en la base de datos Scopus lograda por la UTB. Pues si en el año 2017, se publicaron alrededor de medio centenar de productos $(N=52)$,

6 Recordamos que la base de datos Scopus puede catalogar un mismo producto científico en diferentes áreas de conocimiento, por lo que la suma de estas categorías sería superior al número total de documentos científicos publicados.

7 Adicionalmente se han clasificado las revistas (exclusivamente) en función de los cuartiles asignados por el SCImago Journal Ran, un indicador de impacto basado en las propias métricas de Scopus (Grupo SCImago, 2007) con un reconocimiento internacional y un amplio uso en América Latina (Zacca-González, ChinchillaRodríguez, Vargas-Quesada y De Moya-Anegón, 2014). 
un año después fueron 88; superándose la barrera del centenar y habiéndose alcanzado la cifra de 134 documentos en el año 2019. Un aumento porcentual del 157,69\% respecto el año 2017 y de un 52,27\% con relación al 2018; mientras que el incremento entre los dos primeros años de la serie fue de un 69,23\% (una situación no atribuible al PMA por no estar éste en vigor).

De todas maneras, esta tendencia ascendente quedaría enfatizada al comparar la producción científica total con el resto de las instituciones educativas de la ciudad ya mencionadas (Tabla 1). Mientras que en el año 2019 la principal universidad de la ciudad (la Universidad de Cartagena) aumentó su producción científica con seis nuevos productos respecto el 2018 y la C.U. Rafael Núñez publicó cuatro papers más ese año que el anterior y el resto de las entidades revisadas disminuyeron su cantidad investigativa; la UTB tuvo 46 documentos científicos más que en el anterior período. Esto significaría que esta entidad logró su segundo crecimiento consecutivo, superior al 50\%, en investigación de alto impacto en contraste con el estancamiento o descenso de similares corporaciones educativas de su entorno.

Tabla 1. Producción científica de las principales universidades de Cartagena (2017-2019)

\begin{tabular}{|l|c|c|c|c|}
\hline \multicolumn{1}{|c|}{$\begin{array}{c}\text { Institución educativa superior } \\
\text { de Cartagena de Indias }\end{array}$} & \multicolumn{3}{|c|}{ Período temporal } & $\begin{array}{c}\text { Cambio (en \%) } \\
\mathbf{2 0 1 7 - 2 0 1 9} / \text { 2018- } \\
\mathbf{2 0 1 9}\end{array}$ \\
\cline { 2 - 5 } & $\mathbf{2 0 1 7}$ & $\mathbf{2 0 1 8}$ & $\mathbf{2 0 1 9}$ & \\
\hline Universidad de Cartagena & 206 & 262 & 268 & $+30,09 \% /+2,29 \%$ \\
\hline $\begin{array}{l}\text { Universidad Tecnológica de } \\
\text { Bolívar }\end{array}$ & 52 & 88 & 134 & $+157,69 \% /+52,27 \%$ \\
\hline F.U. Tecnológico Comfenalco & 35 & 27 & 16 & $-54,28 \% /-40,74 \%$ \\
\hline Universidad San Buenaventura & 8 & 8 & 6 & $-25 \% /-25 \%$ \\
\hline C.U. Rafael Núñez & 7 & 15 & 19 & $+171,42 \% /+26,66 \%$ \\
\hline
\end{tabular}

Fuente: elaboración propia con datos obtenidos de Scopus (junio 2020)

Sin embargo, estas cifras positivas respecto a épocas pasadas (274 productos en ese trienio) no lo serían tanto si la producción científica en Scopus de la UTB es analizada de forma más detallada. Al delimitar nuestra búsqueda en función del tipo de producto publicado, se ha observado un incremento desmedido en el número de conference proceedings. Se ha pasado de 14 congresos indexados (frente a 29 artículos/revisiones y 9 libros y capítulos) en el año 2017 (siendo el 26,92\% de la investigación), a 36 actas de estos eventos (en contraste a los 41 artículos/revisiones y 11 libros/capítulos) el año siguiente (un 40,90\%) y a los 53 congresos del año 2019 (un 39,55\%). Si volvemos a comparar estos datos con la institución pública de la 
ciudad (la Universidad de Cartagena, UDC), se evidencia la gran dependencia de este tipo de productos para la UTB.

Mientras que para la UTB el porcentaje de conference proceedings fue del 37,59\% del total (140 journals, 103 conferences, 29 book series y 2 libros), en esos tres mismos años, en la UDC se consiguieron publicar 736 documentos académicos en Scopus, de los cuales tan solo 39 fueron actas de congresos (un 5,29\% del total), siendo la mayoría de ellos artículos científicos de revistas $(\mathrm{N}=682)^{8}$.

Estos datos concordarían plenamente con el ser de la propia UTB, ya que esta institución se ha destacado históricamente por sus programas de ingeniería. Por ende, es obvio que la investigación de esta institución se ha enfocado principalmente en el ámbito de las ciencias puras. Así pues, en el período 2017-2019 la UTB tuvo 133 productos científicos de ingeniería, 88 de ciencias computacionales, 68 de matemáticas, 44 de energía, 43 de física y astronomía y 32 en ciencias sociales; siendo éste el campo ajeno a esta temática con mayor peso del total. Respecto a la evolución anual de estas áreas, considerando la producción científica de ciencias sociales como un arbitrario punto de corte (como límite de temática con producción relevante), se ha identificado una mayor dependencia de la ingeniería respecto el resto de los ámbitos revisados. Una situación que se ha profundizado con el paso del tiempo (Tabla 2).

Tabla 2. Evolución anual de la producción científica de la UTB en las áreas de conocimiento más destacadas (2017-2019)

\begin{tabular}{|l|c|c|c|}
\hline \multirow{2}{*}{$\begin{array}{c}\text { Área de } \\
\text { Conocimiento }\end{array}$} & \multicolumn{3}{|c|}{ Período temporal y mejora anual (en \%) } \\
\cline { 2 - 4 } & $\mathbf{2 0 1 7}$ & $\mathbf{2 0 1 8}$ & $\mathbf{2 0 1 9}$ \\
\hline Ingeniería & 20 & $49(+145 \%)$ & $64(+30,61 \%)$ \\
\hline $\begin{array}{l}\text { Ciencias } \\
\text { computacionales }\end{array}$ & 16 & $29(+81,25 \%)$ & $43(+48,27 \%)$ \\
\hline Matemáticas & 12 & $30(+150 \%)$ & $26(-13,33 \%)$ \\
\hline Energía & - & 22 & $21(-4,54 \%)$ \\
\hline Física y astronomía & 11 & $16(+45,45 \%)$ & - \\
\hline Ciencias sociales & 10 & $12(+20 \%)$ & $21(+75 \%)$ \\
\hline
\end{tabular}

Fuente: elaboración propia con datos obtenidos de Scopus (junio 2020)

Por consiguiente, se puedo afirmar que la investigación de alto impacto de la UTB ha tenido un significativo crecimiento durante los años 2017-2019, además de destacar el excelso trabajo de la Facultad de Ingeniería (más de dos tercios del total) en contraste a la más limitada producción científica del resto de facultades, algo evidenciado también por el idioma usado para escribir los papers, pues el inglés ( $\mathrm{N}$

8 A nuestro parecer estos últimos productos son un claro indicio de mayor calidad que los conference proceedings ya que estos documentos son menos citados que los artículos científicos (Lisée, Larivière y Archambault, 2008), y particularmente se asocian a áreas del conocimiento humano como la ingeniería y la computación siendo casi inexistentes en las ciencias sociales (Lisée et al., 2008). La función fundamental de los congresos es dar a conocer a la comunidad científica los primeros resultados de una investigación (Meyriat, 1966), para finalmente publicar las evidencias finales en una revista, un capítulo de libro o un libro. 
$=240)$ es el lenguaje predominante en ciencias puras mientras que el español $(\mathrm{N}=$ 36) lo es en ciencias sociales y humanidades.

Más específicamente la labor investigativa de los ingenieros se ha nutrido estos últimos años en departamentos muy específicos de esa facultad (Tabla 3); a destacar los programas de ingeniería eléctrica y electrónica en los que han sobresalido investigadores como el Dr. Óscar Montoya (perteneciente a los dos anteriores programas e incorporado a la UTB en el año 2018). Tan solo este autor individual ha publicado, por medio de equipos interdisciplinares, 64 documentos en dos años, lo que equivale al $23,35 \%$ de la producción científica de esta universidad en el período temporal estudiado; indicándose a continuación los investigadores más prolíficos de esta entidad y la facultad y el programa al que pertenecen.

Tabla 3. Principales docentes investigadores de la UTB (2017-2019)

\begin{tabular}{|c|c|c|c|c|}
\hline \multirow[t]{2}{*}{$\begin{array}{c}\text { Investigador de la UTB } \\
\text { y nivel académico }\end{array}$} & \multirow[t]{2}{*}{$\begin{array}{c}\text { Facultad y programa de } \\
\text { pertenencia }\end{array}$} & \multicolumn{3}{|c|}{$\begin{array}{c}\text { Producción científica en } \\
\text { la UTB }\end{array}$} \\
\hline & & 2017 & 2018 & 2019 \\
\hline Óscar Montoya (PhD.) & $\begin{array}{c}\text { Fac. Ingeniería / Pr. Ing. } \\
\text { eléctrica, Pr. Ing. electrónica }\end{array}$ & - & 29 & 44 \\
\hline Andrés Marrugo (PhD.) & $\begin{array}{c}\text { Fac. Ingeniería / Pr. Ing. } \\
\text { mecánica, Pr. Ing. Mecatrónica }\end{array}$ & 3 & 9 & 11 \\
\hline Sonia Contreras (PhD.) & $\begin{array}{c}\text { Fac. Ingeniería / Pr. Ing. } \\
\text { eléctrica, Pr. Ing. electrónica }\end{array}$ & 9 & 3 & 6 \\
\hline José Luis Villa (PhD.) & $\begin{array}{c}\text { Fac. Ingeniería / Pr. Ing. } \\
\text { eléctrica }\end{array}$ & 5 & 5 & 6 \\
\hline Lenny Romero (PhD.) & Facultad de Ciencias básicas & 1 & 6 & 7 \\
\hline & $\ldots$ & & & \\
\hline Karol Gutiérrez (PhD.) & $\begin{array}{l}\text { Fac. CC.SS. y humanidades / } \\
\text { Pr. Psicología }\end{array}$ & 1 & 1 & 3 \\
\hline Pedro Vázquez (PhD.) & $\begin{array}{l}\text { Fac. CC.SS. y humanidades / } \\
\text { Pr. Psicología }\end{array}$ & 2 & 1 & 2 \\
\hline
\end{tabular}

Fuente: elaboración propia con datos de Scopus (junio 2020) y el directorio de la UTB

En función de la calidad de las publicaciones alcanzadas (habiéndonos ceñido en exclusiva a las revistas indexadas en SCImago (SJR) ${ }^{9}$ más populares donde los docentes de la UTB, como mínimo, han publicado dos veces o más en un año), las estadísticas de los años 2017-2019 han reflejado no solo una mejoría de la cantidad de la investigación. En el 2017 tan solo destacaban las revistas Communications in Computer and Information Science (Q3 en el año 2017) con 7 colaboraciones, Óptica Pura y Aplicada (también en Q3) con 2 publicaciones y Water (Switzerland) (cuartil

9 Siempre se indicó el cuartil más elevado de la revista en caso de que la publicación fuera catalogada en dos áreas o más de conocimiento por SCImago. 
Q1) con otras 2 aportaciones. Lamentablemente la aparición de colaboraciones en revistas depredadoras, como Espacios $^{10}$ (con 4 publicaciones) ya era una realidad, una práctica que se mantendría con el paso de los siguientes años.

En el año 2018, los profesores de la UTB siguieron publicando en las anteriores revistas [Communications in Computer and Information Science con nueve trabajos (Q3 en el año 2018) y Óptica Pura y Aplicada con tres documentos (Q3 en el año 2018)] y se incorporaron en este cómputo Journal of Energy Storage (Q1, 2018) con cinco papers, Información Tecnológica (Q3, 2018) con dos trabajos y finalmente Urban Water Journal (Q1, 2018) con otro par de informes. La presencia de la malograda revista Espacios tendría esta vez solo una representación.

El último año de la serie que fue analizado, 11 revistas (solo una de ellas perteneciente al área de las ciencias sociales) tuvieron dos o más colaboraciones de docentes de la UTB habiendo en todo el conjunto solo una revista depredadora (Ibima Business Review) con un paper. Además de las revistas ya conocidas como Communications in Computer and Information Science (Q3, 2019), manteniendo ésta una imparable progresión ascendente con 11 artículos, Información Tecnológica (Q3 / tres trabajos), Water (Switzerland) (Q1, dos trabajos) y Journal of Energy Storage (Q1, dos estudios), las publicaciones más destacables fueron las siguientes:

- Electric Power Systems Research (Q1): tres artículos.

- Advances in Electrical and Electronic Engineering (Q3): dos artículos.

- Ain Shams Engineering Journal (Q1): dos artículos.

- International Journal of Electrical Power and Energy Systems (Q1): dos trabajos.

- Journal of Hydraulic Research (Q1): dos trabajos.

- Journal of Transport and Health (Q1): dos trabajos.

- Current Psychology (Q2): dos papers.

\section{Discusión de resultados y conclusiones}

Conocida la tendencia ascendente en la cantidad de la producción científica de la UTB que fue revisada durante los años 2017-2019, vista la comparación de otras instituciones de su entorno, el aumento de la calidad de las revistas más manejadas por los investigadores a la hora de dar a conocer a la comunidad sus hallazgos, junto las perspectivas halagüeñas en el área investigativa para el año 2020 y la gran cantidad de docentes de esta universidad que lograron su primera publicación en Scopus en el 2019; se ha asumido que el PMA de la UTB contribuyó significativamente a la mejora de la investigación de este centro educativo superior de la ciudad de Cartagena de Indias.

10 A pesar de que esta publicación no está en la lista Beall’s list, el instrumento más reconocido para identificar a este tipo de journals (Somoza-Fernández, Rodríguez-Gairín y Urbano, 2016), por sus características intrínsecas la susodicha revista se ha catalogado de esta manera, como lo ha considerado Cortés-Sánchez (2019), entre otros autores. 
Se debe incidir en la idea de que si la subida (considerable) de la cantidad de documentos investigativos de alto impacto de la UTB durante los años 2017-2018 se ha debido a aspectos exógenos al PMA por no existir éste aún (como la contratación de profesores en el año 2018 que fueron muy prolíficos a la hora de conseguir publicaciones), existiendo un entorno local favorable a esta tendencia ya que la UDC también tuvo un comportamiento similar en ese mismo período; consideramos, en definitiva, que esta propuesta evaluativa fomentó que el crecimiento científico de la UTB se mantuviera alto en comparación con el estancamiento de su competidor público y otras universidades de menor tamaño.

La parte negativa de esta situación es que el PMA enfatizó algunas debilidades de este rápido crecimiento logrado, ya que en el año 2019 se hizo evidente la específica hiperdependencia en investigación que tiene esta institución educativa respecto a un número muy reducido de docentes que poseen unas características comunes. Dentro de los elementos identificables de estos profesionales, destacaríamos, además de su capacidad de trabajo y organización, su formación (doctoral) en ingeniería, especialmente el campo de la electrónica y la electricidad, la preferencia por trabajar siempre con un equipo de trabajo cerrado ya consolidado, el saber identificar nítidamente las revistas objetivo de su sector y el publicar de manera masiva en conference proceedings $^{11}$ (en comparación con otras instituciones) como antesala de los propios artículos científicos, es lo que explicaría unas tasas de eficiencia investigativa tan elevadas.

Es evidente por tanto que, en el supuesto futuro de una rotación laboral de ciertos programas de ingeniería de esta institución educativa, esto afectaría duramente a la capacidad investigativa de esta universidad. Se requiere por tanto consolidar otro tipo de programas de ingeniería para posteriormente fortalecer el área investigativa de la UTB de las demás facultades; teniendo, desde nuestra experiencia subjetiva (como investigadores y habiendo conversado ampliamente con colegas de otras áreas) la plena consciencia que los tiempos editoriales del campo de las ciencias sociales y las humanidades son mucho más largos que en las ingenierías, las ciencias puras y las ciencias de la salud.

Uno de los aspectos más negativos de la implementación del PMA es la sensación de una gran parte del profesorado en sentirse evaluado por situaciones laborales que son completamente ajenas a su control (como por ejemplo el tiempo de respuesta de una revista científica); algo que ha producido inconformidad dentro de este gremio, más teniendo en cuenta que con el diseño actual de ese PMA pareciera irrelevante el cómo se dan las clases magistrales u otros logros realizados por el profesor (creación de eventos, diseño y realización de tareas promocionales, visitas culturales a departamentos públicos, fortalecimiento de los lazos con las empresas...) ya que estas actividades no fueron recogidas en el citado documento evaluativo.

Es por ello por lo que, como recomendación final hacia la búsqueda de la excelencia universitaria, se ha planteado brevemente una mejora del PMA de la UTB

11 Creemos que la estructura del PMA de la UTB, al no diferenciar entre artículos científicos, libros, capítulos de libro y conference proceedings, ha fomentado la elaboración de este último tipo de productos. 
(Anexo), específicamente para el apartado de investigación; viendo nosotros oportuno el hacer las siguientes modificaciones al mismo:

- Modificar las puntuaciones dadas a los trabajos publicados en Scopus y WoS. Se le daría una mayor relevancia a los libros y a los artículos de cuartil Q1 y Q2 en SCImago (valor x2) respecto a los dos cuartiles restantes y a los capítulos de libro (valor $\mathrm{x} 1$ ), siendo los conference proceedings de menor rango para fomentar la investigación en el resto de las categorías (valor 1/2).

- Valorar mínimamente las publicaciones de otras bases de datos como Publindex, SciELO o ESCI (entre otras) junto al resto de producción científica (libros, cartillas pedagógicas...) en el caso de que éstas no estén indexadas en Scopus (valor $1 / 4$ ). El asumir que estos estudios no sirven para cumplir el PMA producirá un abandono de estas labores y es básico que una universidad investigue sobre aspectos locales de su entorno, así sus resultados no sirvan para conseguir métricas en Scopus o WoS.

- Crear objetivos de publicación diferenciados por facultad haciendo menos exhaustiva esta meta para las áreas que no son de ingeniería debido al fuerte desnivel existente en la capacidad y formación investigativa de los profesores de esta institución.

- Penalizar severamente a los docentes que publiquen en revistas y congresos depredadores (valor x0).

\section{Referencias bibliográficas}

Alarcón-Quinapanta, M. R., Freire-Lescano, L. R., Pérez-Barral, O., Frías-Jiménez, R. A. y Nogueira-Rivera, D. (2019). Medición del rendimiento del talento humano en instituciones de educación superior: producción científica. Ingeniería industrial, 40 (1), 24-36.

Arango-Soto, D. (2010). La primera universidad del Caribe colombiano. Un modelo ilustrado para América Colonial. Desafíos, 10, 8-43.

Bayona-Rodríguez, H., Bedoya, J. G. y Sánchez, F. J. (2018). Eficiencia de la producción científica de las universidades colombianas. Documento CEDE, 36.

Caldera-Serrano, J. (2018). Repositorios públicos frente a la mercantilización de la ciencia: apostando por la ciencia abierta y la evaluación cualitativa”. Métodos de Información, 9 (17), 74-101. doi: https://dx.doi.org/10.5557/IIMEI9-N17-074101

Castillo, J. J. y Moré, P. (2016). Por una sociología del trabajo académico. La precarización del trabajo de enseñar e investigar en la universidad. Sociología del Trabajo, 88, 7-26.

Cortés-Sánchez, J. D. (2019). Innovation in Latin America through the lens of bibliometrics: crammed and fading away. Scientometrics, 121, 869-895. doi: https://doi.org/10.1007/s11192-019-03201-0

Ferro-Bayona, J. y Amarís, M. (1991). En los 25 años de la Universidad del Norte: orígenes y fundaciones de universidades costeñas. Huellas, 32, 5-21.

Giménez-Toledo, E. (2014). Imposturas en el ecosistema de la publicación científica. Revista de Investigación Educativa, 32 (1), 13-23.

Gómez-Velasco, N., Jiménez-González, A., Rodríguez-Gutiérrez, J. y Romero-Torres, M. (2020). Comparación de la eficiencia científica entre Colombia y México a través de indicadores relativos de producción y calidad científica. Revista Española de Documentación Científica, 43 (2). doi: https://doi.org/10.3989/redc.2020.2.1644 
Grupo SCImago (2007). SCImago Journal \& Country Rank: un nuevo portal, dos nuevos rankings. El Profesional de la Información, 16, 645-646.

Lisée, C., Larivière, V. y Archambault, E. (2008). Conference proceedings as a source of scientific information: A bibliometric analysis. Journal of the American Society for Information Science and Technology, 59 (11). doi: https://doi.org/10.1002/asi.20888

Martínez-Dueñas, W. A. y Arias-Ocampo, A. M. (2017). Editorial. Jangwa Pana, el Caribe y -Publish or perish-. Jangwa Pana, 16 (1), 9-26.

Maz-Machado, A., Jiménez-Fanjul, N. N. y Villarraga, M. E. (2016). La producción científica colombiana en SciELO: un análisis bibliométrico. Revista Interamericana de Bibliotecología, 39 (2), 111-119.

Mongeon, P. y Paul-Hus, A. (2016). The journal coverage of Web of Science and Scopus: a comparative analysis. Scientometrics, 106, 213-228. doi: https://doi.org/10.1007/s11192015-1765-5

Montes, I. C. y Mendoza, P. (2018). Docencia e investigación en Colombia desde la perspectiva del capitalismo académico. Archivos analíticos de políticas educativas, 26 (40). doi: http://dx.doi.org/10.14507/epaa.26.3220

Meyriat, J. (1966). La información científica en las ciencias del hombre: Su circulación. Revista Mexicana de Sociología, 28 (4), 929-938.

Muñoz-Ñungo, B., Rodríguez-Faneca, C. y Gutiérrez-Rubio, D. (2020). La investigación en educación matemática en Emering Sources Citation Index (ESCI): la producción de Colombia. Educación y Sociedad, 3 (1), 1-11.

Puentes-Cala, M. (2019). Bases de datos con ánimo de lucro y la mercantilización de las publicaciones científicas. Colombia, una vía de entrada. E-Ciencias de la Información, 9 (2). doi: http://dx.doi.org/10.15517/ECI.V9I2.37498

Rodríguez, E., Naranjo, S. y González, D. L. (2015). Publindex: más que un proceso de indexación. Revista El Ágora USB, 15 (1), 29-41.

Rueda-Barrios, G. y Rodenes-Adam, M. (2016). Factores determinantes en la producción científica de los grupos de investigación en Colombia. Revista Española de Documentación Científica, 39 (1). doi: http://dx.doi.org/10.3989/redc.216.1.1198

Ripoll, M. T. (2011). La historia de la UTB. Cartagena de Indias: Ediciones Unitecnológica.

Rodríguez-Morales, A. J., Culquichicón-Sánchez, C. y Gil-Restrepo, A. F. (2016). Baja producción científica de decanos en facultades de medicina y salud de Colombia: ¿una realidad común en Latinoamérica? Salud Pública de México, 58 (4). doi: https://doi.org/ 10.21149/spm.v588i4.7809

Santa, S. y Herrero-Solana, V. (2010). Producción científica de América Latina y el Caribe: una aproximación a través de los datos de Scopus (1996-2007). Revista Interamericana de Bibliotecología, 33 (2), 379-400.

Somoza-Fernández, M., Rodríguez-Gairín, J. M. y Urbano, C. (2016). Presence of alleged predatory journals in bibliographic databases: analysis of Beall's list. El Profesional de la Información, 25 (5), 730-737. doi: https://doi.org/10.3145/epi.2016.sep.03

Suárez-Ortega, M., García-Mingo, E. y Ruiz-San Román, J. A. (2012). When Español is not enough: research, write, translate and publis or... perish. International Journal of Leadership in Education, 15 (4), 463-482. doi: https://doi.org/10.1080/13603124. 2012.696709

Torres-Samuel, M., Vásquez, C. L., Viloria, A., Varela, N., Hernández-Fernández, L. y Portillo-Medina, R. (2018). Analysis of Patterns in the University World Rankings Webometrics, Shangai, QS and SIR-SCimago: Case Latin America. En Y. Tan, Y. Shi y Q. Tang (eds.). Data Mining and Big Data. DMBD 2018. Lecture notes in Computer Science. Cham: Springer. doi: https://doi.org/10.1007/978-3-319-93803-5_18 
Vélez-Cuartas, G., Lucio-Arias, D. y Leydesdorff, L. (2016). Regional and global science: Publications from Latin America and the Caribbean in the SciELO Citation Index and the Web of Science. El Profesional de la Información, 25 (1), 35-46. doi: http://dx.doi.org/10.3145/epi.2016.ene.05

Viloria-De La Hoz, J. (2006). Educación Superior en el Caribe Colombiano: Análisis de Cobertura y Calidad. Documentos de Trabajo Sobre Economía Regional, 69.

Zacca-González, G., Chinchilla-Rodríguez, Z., Vargas-Quesada, B. y De Moya-Anegón, F. (2014). Bibliometric analysis of regional Latin America 's scientific output in Public Health through SCImago Journal \& Country Rank. BMC Public Health, 14 (632). doi: https://doi.org/10.1186/1471-2458-14-632

\section{Anexo}

Plan de Metas Anual (en el área de Investigación y Producción Intelectual) de la Universidad Tecnológica de Bolívar (2019)

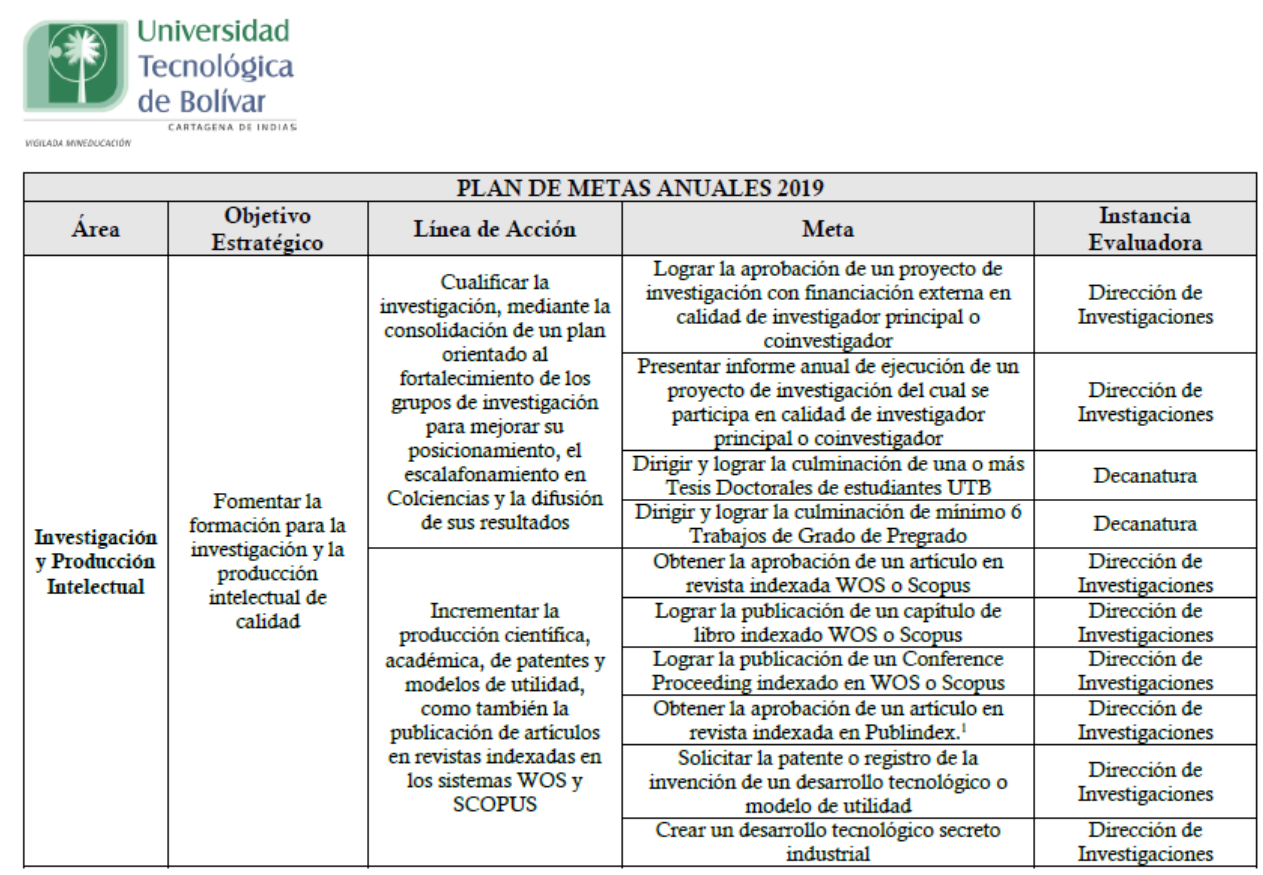

\footnotetext{
${ }^{1}$ Aplica exclusivamente para profesores cuyo máximo nivel de estudios es la Especialización.
} 